\title{
Three-Dimensional Reconstruction of Wafer Solder Bumps using Binary Pattern Projection
}

\author{
"Jun Cheng, "Ronald Chung, "*Edmund Y. Lam, "Kenneth S.M. Fung, "Fan Wang, "WH Leung \\ *Dept. Automation \& Comp-Aided Engr., \\ The Chinese Univ. of Hong Kong, \\ Hong Kong, CHINA \\ ** Department of EEE, \\ ${ }^{\#}$ ASM Assembly \\ The Univ. of Hong Kong, Automation Ltd., \\ Hong Kong, CHINA Hong Kong, CHINA
}

E-mail:\{jcheng,rchung\}@acae.cuhk.edu.hk

\begin{abstract}
As the electronic industry advances rapidly, the shrunk dimension of the device leads to more stringent requirement on process control and quality assurance. For instance, the tiny size of the solder bumps grown on wafers for direct die-todie bonding pose great challenge to the inspection of the bumps' 3D quality. Traditional pattern projection method of recovering $3 \mathrm{D}$ is about projecting a light pattern to the inspected surface and imaging the illuminated surface from one or more points of view. However, image saturation and the specular nature of the bump surface are issues. This paper proposes a new 3D reconstruction mechanism for inspecting the surface of such wafer bumps. It is still based upon the light pattern projection framework, but uses the Ronchi pattern - a pattern that contrasts with the traditionally used gray level one. With the use of a parallel or point light source in combination with a binary grating, it allows a discrete pattern to be projected onto the inspected surface. As the projected pattern is binary, the image information is binary as well. With such a bright-or-dark world for each image position, the above-mentioned difficult issues are avoided. Preliminary study shows that the mechanism holds promises that existing approaches do not.
\end{abstract}

Keywords: Three-Dimensional Reconstruction, Binary Pattern Projection, Ronchi Pattern, Wafer Bump, Codeword, Specular surface.

\section{INTRODUCTION}

As the electronic industry advances rapidly toward manufacturing smaller, lighter, faster, and cheaper products, area array packages including BGA, CSP, flip chips, wafer bumping and wafer-level packaging (WLP) quickly become the focus of IC packaging technology. They could help improve device performance and manufacturability, and ultimately reduce cost.

However, the shrunk dimension of the device also leads to more stringent requirement on process control and quality assurance. A particular challenge is the advancement of vision technique for inspecting the 3D surface of wafer bump accurately and efficiently. Specifically, bump heights need to be measured and examined in 3D to see if they are defective. However, the tiny size of the wafer bumps - which are typically of diameter 60 microns or so - make traditional technologies for inspecting PCB solder bumps not applicable.

There have been a few inspection mechanisms proposed for wafer bumps: laser triangulation[5,13,14], confocal microscopy[4,6,7,10,12], and gray-level pattern projection[1,2,3,8,9,11]. However, laser triangulation is of low speed and low resolution, confocal microscopy involves moving parts and has limitation in its operation speed, and gray-level pattern projection has the problems of image brightness saturation and high sensitivity to noise.

Pattern projection method is about projecting a light pattern and capturing image of the illuminated scene from one or more points of view. If the pattern is made binary or discrete, the problems of image brightness saturation and sensitivity to noise could be relieved, since only bright or dark instead of a gray level intensity needs be concerned. By changing the binary pattern a number of times, each time capturing a distinct image of the illuminated surface, one could attach each 
position on the illuminated surface with a binary code in the sequence of captured images. By the use of suitable changes in the pattern, the binary code could even be made unique for each surface position. With such binary codes, position correspondences between the image plane and the projected pattern could be easily established. 3D reconstruction is then possible on these coded points via triangulation.

In traditional binary pattern projection, the illumination pattern is constructed by an array of LCD light sources. However, such arrangement is not suitable for wafer bump inspection. The reason is, for the patterned light to cover a significant area of the surface of each wafer bump (and thus allow it to be reconstructed more fully in 3D), the physical area occupied by the light sources has to span a large space. That is undesirable as it could hinder the operation of the various moving parts involved in wafer processing.

This work aims at coming up with a new 3D reconstruction mechanism that is based upon the Ronchi pattern projection framework but occupies only a small physical area. It aims at achieving an inspection device that is of small size yet capable of reconstructing a large area of each wafer bump. More precisely, we propose to use a parallel or point light source in combination with a Ronchi grating to allow a discrete pattern to be projected onto the inspected surface. As only a single light source is needed the whole system is compact occupying only a small physical size. To change the Ronchi pattern in every image snapshot, the Ronchi pattern will be shifted in space with respect to the stationary light source and inspected surface and camera. As pointed out earlier, since the projected pattern is binary, the image information is binary as well. With such a bright-or-dark world for each image position, issues like image saturation and specular nature of the imaged surface could be avoided. Preliminary study through theoretical analysis and empirical experiments on synthetic and real image data show that the mechanism holds a number of advantages over existing approaches.

The organization of the paper is as follows. In Section 2, we outline how Ronchi pattern projection could help recover $3 \mathrm{D}$ structure. In Section 3 we show some preliminary experimental results that demonstrate the validity of proposed approach. Concluding remarks are presented in Section 4.

\section{RONCHI PATTERN PROJECTION METHOD}

Continuous grating method (e.g., sinusoidal grating method) uses gray-level intensities to determine phase displacement and then surface profile. Because of the dependence on the absolute value of the perceived intensity, it has to tackle problems like brightness saturation, multi-reflections, and noise or disturbance to gray level brightness.

\subsection{Correspondence Establishment}

If the continuous fringe pattern is replaced by Ronchi (i.e., light-passing and light-blocking) pattern, a distinct binary code would be given to each surface point through a number of physical shifting of the fringe grating and imaging of the illuminated surface. Such binary codes are observable in both the fringe grating and the image data, and would allow position correspondences between the fringe grating plane and the image plane be established. Such correspondences, each induced by a surface point on the illuminated surface, would in turn allow the 3D shape of the illuminated surface to be reconstructed.

For simplicity, suppose that the optical axes of the camera and illumination (we define the optical axis of the illumination as the line that contains the source of illumination and is perpendicular to the fringe grating plane) are aligned so that they are co-planar. We shall refer to the plane that contains both optical axes as $\Pi_{P}$. As illustrated by Fig. 1, suppose also that the Ronchi grating is placed so that its plane is parallel to the line $V$ that joins the camera's optical center and the light source, its fringes' direction is perpendicular to plane $\Pi_{P}$, and its motion is in the direction of $V$. When pattern is projected onto the target surface, the surface will appear to have some bright or dark zones.

By shifting the grating a number of times, each time capturing a new image of the target surface using the camera, a binary codeword is developed for each image position over the sequence of captured images. The image position corresponds to a particular position on the target surface and in turn a particular position on the grating plane, and the associated codeword is readable from both the image data and the Ronchi grating. Fig. 2 illustrates the codeword of a particular position on the grating, where a dark fringe corresponds to a " 0 " and a bright fringe a " 1 ". 


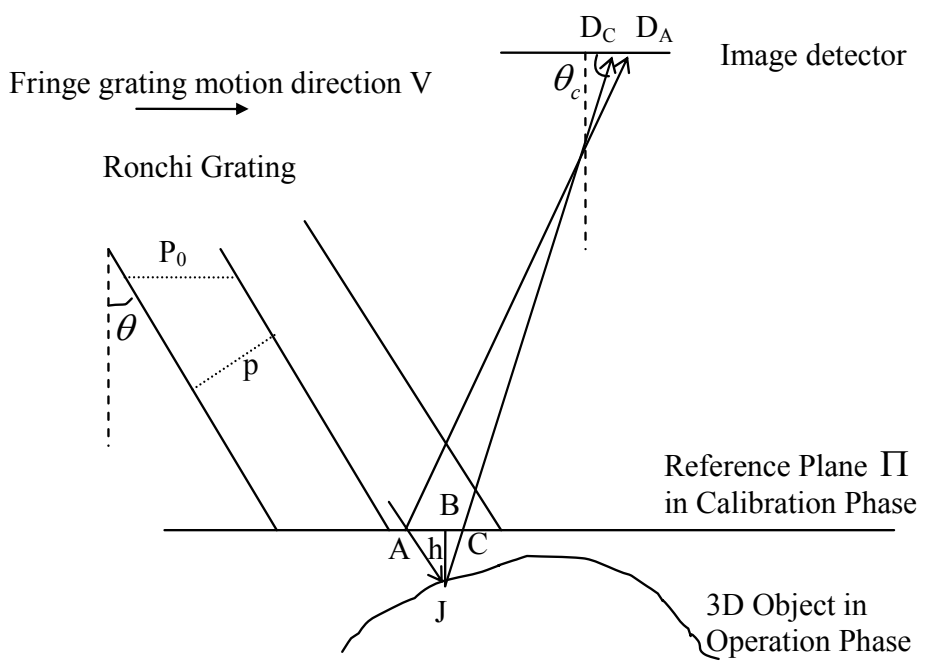

Fig. 1. Optical geometry for measurement.

Positions on the same fringe will have exactly the same codeword, so the fringe grating motion alone only induces lineto-contour correspondences between the grating plane and the image plane. However, correspondence ambiguity within each fringe could be resolved by the use of the epipolar geometry that exists over the camera and the illumination source (on the epipolar geometry, the illumination source is to be regarded as the optical center of a second camera, and the fringe grating plane as the image plane of that camera). By the use of both the codeword and the epipolar geometry, point-to-point correspondences between the grating plane and image plane could ultimately be established.

\subsection{D Measurement}

Consider the system geometry shown in Fig. 1, where a collimated Ronchi pattern of period $p$ is projected, at an offset angle $\theta$ from the imaging direction, onto first a reference plane $\Pi$ and then later a 3D object, where for every image position $(x, y)$ the object shape $h(x, y)$ measured from the reference plane is desired. Here we assume that the reference plane is perpendicular to plane $\Pi_{P}-$ the plane that contains the optical axes of both the camera and the illumination.

Suppose $D_{A}$ is the detector on the image plane that measures the image information associated with point $A$ on the reference plane $\Pi$. Suppose $D_{C}$ is another detector on the image plane that measures the image information at point $C$ on $\Pi$, which happens to be also the detector associated with point $J$ on the object due to the overlap in the projection rays as illustrated in Fig. 1. The binary information sensed at $D_{C}$ is the same as that sensed at $D_{A}$ due to the same pattern projection, so the correspondence between $D_{C}$ and $D_{A}$ could be established by simply observing the Ronchi patterns at the two image positions in the reference plane image data and the object image data respectively.

Suppose $A$ and $J$ are projections of the same Ronchi pattern on the reference plane and the 3D object respectively. Suppose that $A$ is in the $i$ th column of the reference image (i.e., detector $D_{A}$ is in the $i$ th column of the camera CCD array), and $J$ is in the $j$ th column of the object image (i.e., detector $D_{C}$ is in the $j$ th column of the CCD array). 
Suppose also that the CCD resolution is $R_{C C D}$ (in terms of um/pixel) and magnification of imaging system is $M$ (in terms of magnified times). The distance $A C$ can be determined form the separation of $i$ and $j$ in the image plane as:

$$
A C=|i-j| \frac{R_{C C D}}{M}
$$

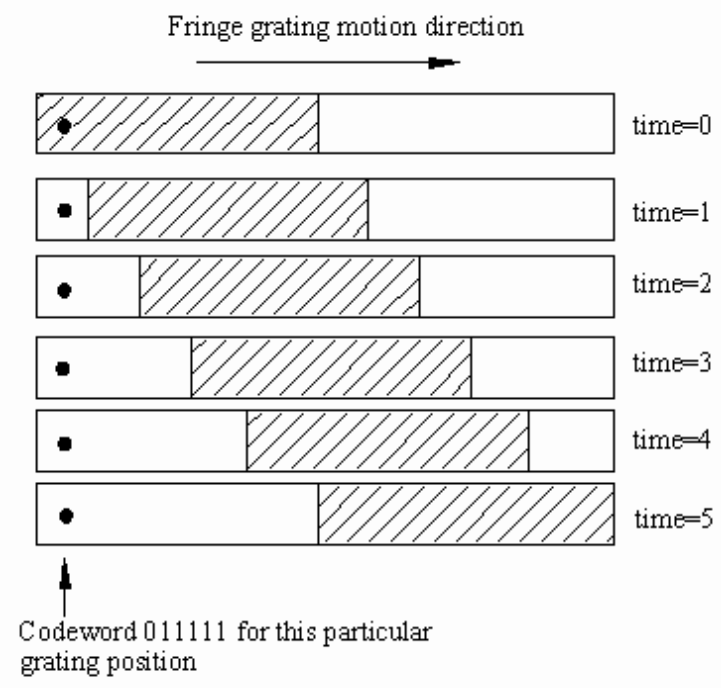

Fig. 2. Codeword production mechanism.

In turn, $A C$ is related to the surface height $h=B J$ by:

$$
h=B J=A C /\left(\tan \theta+\cot \theta_{c}\right)=|i-j| \frac{R_{C C D}}{M} /\left(\tan \theta+\cot \theta_{c}\right)
$$

where the angles $\theta$ and $\theta_{c}$ are as shown in Fig. 1. In practice, since the image sensing array is very small compared with the imaging distance and could be adjusted to have an orientation almost orthogonal to the reference plane, $\theta_{c}$ is nearly 90 degree. The above relation could thus be further simplified to:

$$
h=A C \cdot \cot \theta=|i-j| \frac{R_{C C D}}{M} \cot \theta
$$

The above equation is used to obtain depth.

\section{PRELIMINARY EXPERIMENTS}

We have implemented the proposed system and tested it with micro-objects of size 40 800 microns in the diameter, whose surface reflectivity and size are the same as those of the wafer bumps the system is targeted at. 


\subsection{System Setup}

Calibration should be done first, which ensures that the illumination, the imaging, the grating plane, and the grating motion are aligned properly. The optical axes of the camera and illumination should be on the same plane $\Pi_{P}$. The Ronchi grating should have its plane parallel to the line $B$ that joins the camera's optical center and the light source, its fringes' direction perpendicular to plane $\Pi_{P}$, and its motion in the direction of $B$. It is also required that the reference plane be positioned so that it is perpendicular to plane $\Pi_{P}$.

A Ronchi grating with period 200 microns and an incident angle $\theta=30^{\circ}$ of projection was adopted. The projection system consisted of an illuminator and several sets of lens. The grating was shifted in space 5 times, each time by 20 microns. The magnification of the projection system was 3 times, which means the projected pattern would be enlarged 3 times to have a period of 600 microns on the inspected surface. The imaging system had a magnification of 0.75 times, a resolution of $1000 \times 1000$, and a pixel size of 7.4 micron $\times 7.4$ micron in the CCD sensing array.

\subsection{Operation Details}

In the calibration phase, we projected the fringe pattern onto a sheet of white paper, which serves as the reference plane П. By this step, a codeword for each position on the reference plane $\Pi$ was transferred from the corresponding position on the grating plane. Depth determination processing could thus be transferred from over the grating-and-image planepair to over the reference-and-image plane-pair, through the use of Equation (3) above. The image sequence for the reference plane is shown in Fig. 3.

In the operation phase, we projected the same fringe pattern onto the target object. With 5 shifting of the pattern and imaging of the illuminated object, we established also a codeword for each point on the inspected surface. To put simply, the codeword is the sequence of " 1 "s and " 0 "s in the image sequence (shown in Fig. 4) at every image position.

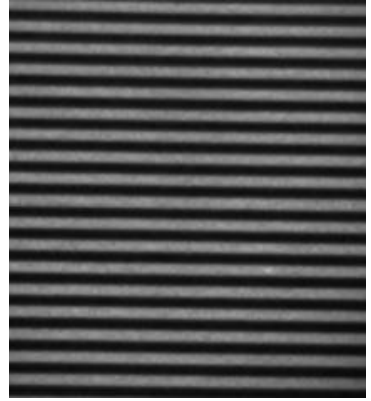

1

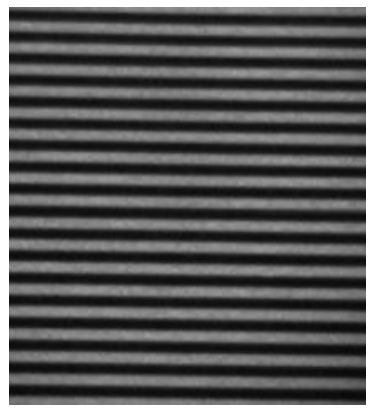

4

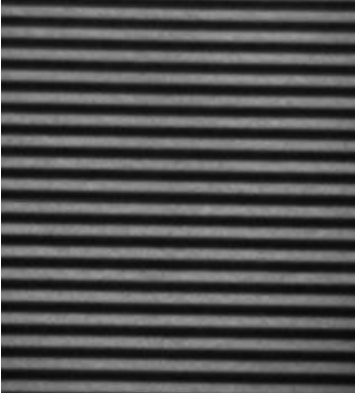

2

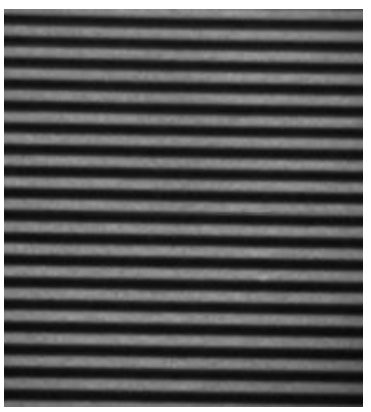

5

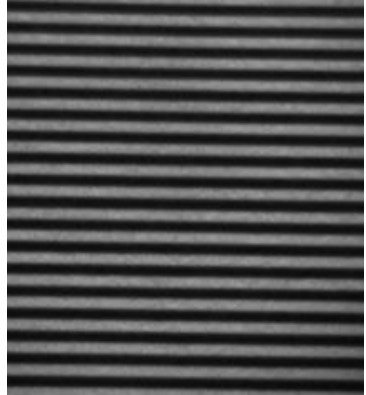

3

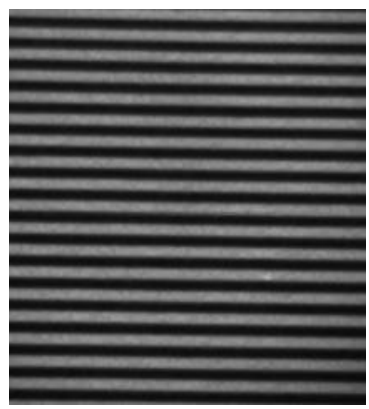

6

Fig. 3. Image sequence of the reference plane in the calibration phase under Ronchi pattern projection. 


\subsection{Result}

Since the wafer surface is made of metal solder and substrate, the surface reflectivity on it could vary from being specular to being diffuse. This leads to difficulty in converting the captured gray level image to the expected binary image, as observed from Fig. 4. Traditional methods of binarizing an image could cause much error. We use a novel approach for intensity binarization on inspected surface with uneven reflectivity. By the use of a novel edge detection mechanism on the spatial-temporal volume of the associated image data, the gray-level images can be converted to binary image. From the binarized image sequence, we obtain the codeword maps (shown in Fig. 5 and 6 , where different colors represent different codewords) for the reference plane and the inspected surface respectively. With the two codeword maps, corresponding point pairs in the two maps could be obtained by associating image points with the same sequence of " 1 "s and " 0 "s. With the correspondences, 3D position or depth disparity could be estimated for each point of the inspected surface using the method outlined in Section II. Fig. 7 shows the 3D reconstruction of the inspected surface.

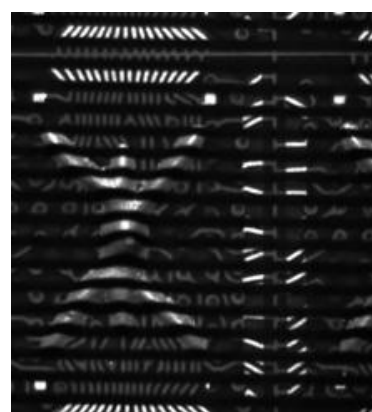

1

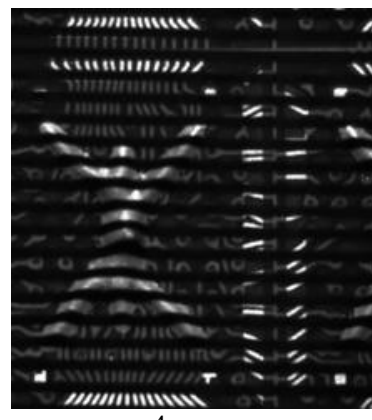

4

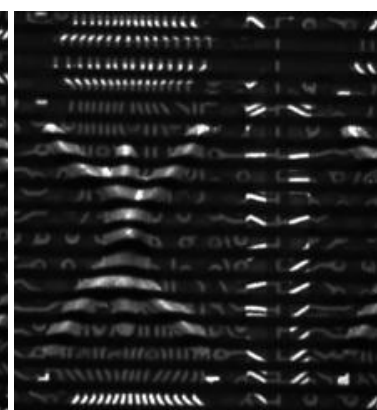

2

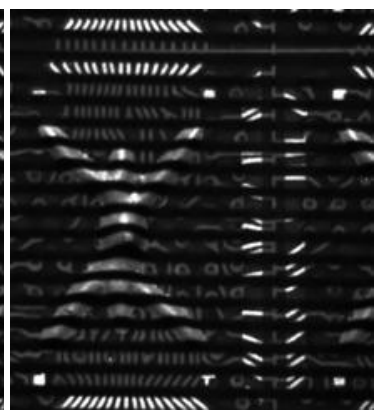

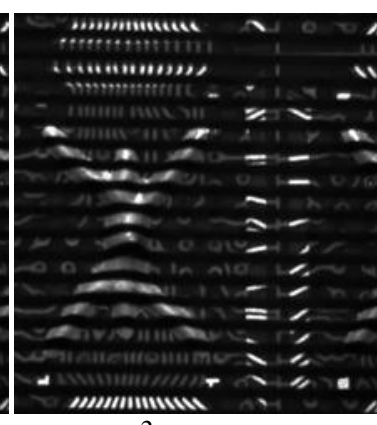

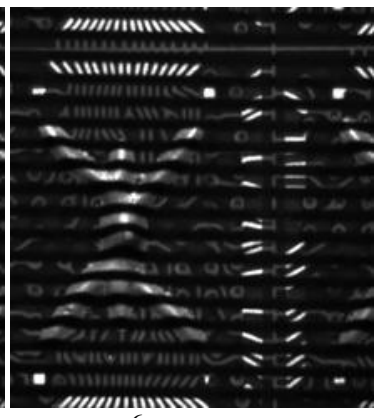

Fig. 4. Image sequence of the inspected surface in the operation phase under projection of the same pattern.

We would like to point out that in our current implementation we only consider image points that are close to the edge of the Ronchi pattern in at least one of the captured images, and attempt correspondence extraction and 3D reconstruction at those points only. The reason is, for other (non-edge) places in the image, neighboring image positions might have similar or the same codeword due to the limited image resolution, and that would compromise the accuracy in their $3 \mathrm{D}$ reconstruction. We found from our experiments that image positions that are edge points in either one of the captured images constitute about $50 \%$ of all the image positions, and at such positions depth could be recovered directly. As for the $3 \mathrm{D}$ information of the other $50 \%$ of the image positions, we suggest that they be interpolated from those of the edge points. 


\section{CONCLUSION AND FUTURE WORK}

A new approach for reconstructing wafer bumps has been presented. Empirical analyses show that the approach could effectively reconstruct $3 \mathrm{D}$ for even very small objects. Because of its discrete nature, the approach has a number of advantages over existing methods: it suffers less from (1) image brightness saturation; (2) specular nature of the solder surface, and (3) effect of noise to image data. Future work includes 3D reconstruction for non-edge points in the image data, optimal design of the grating, and extension of the approach for tackling the problem of multiple reflections.

\section{ACKNOWLEDGMENT}

The work described in this paper was substantially supported by a grant from the Innovation and Technology Commission of Hong Kong Special Administrative Region, China, under an Innovation and Technology Fund with Project Code UIM/111.

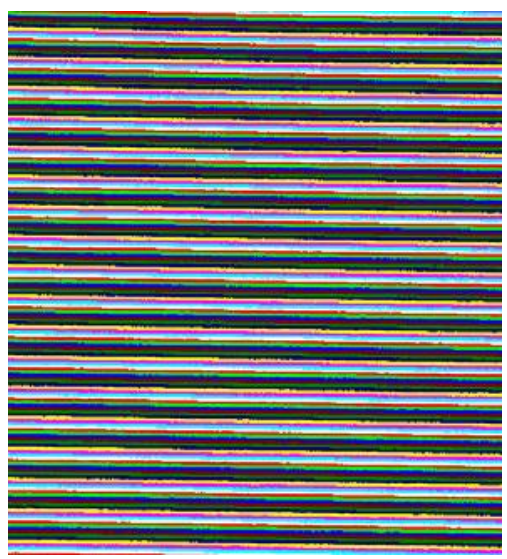

Fig. 5. Codeword Map for Reference Plane.

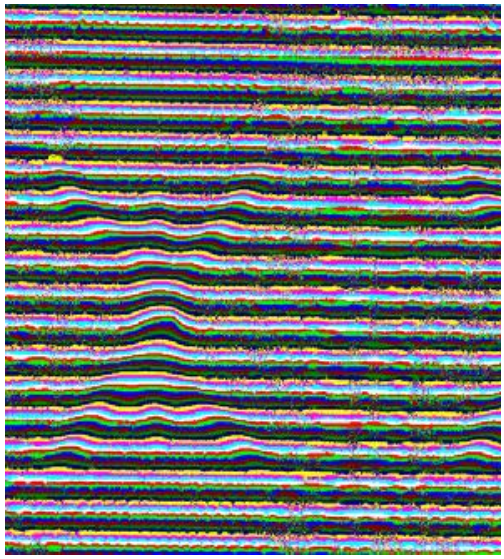

Fig. 6. Codeword Map for Inspected Surface. 

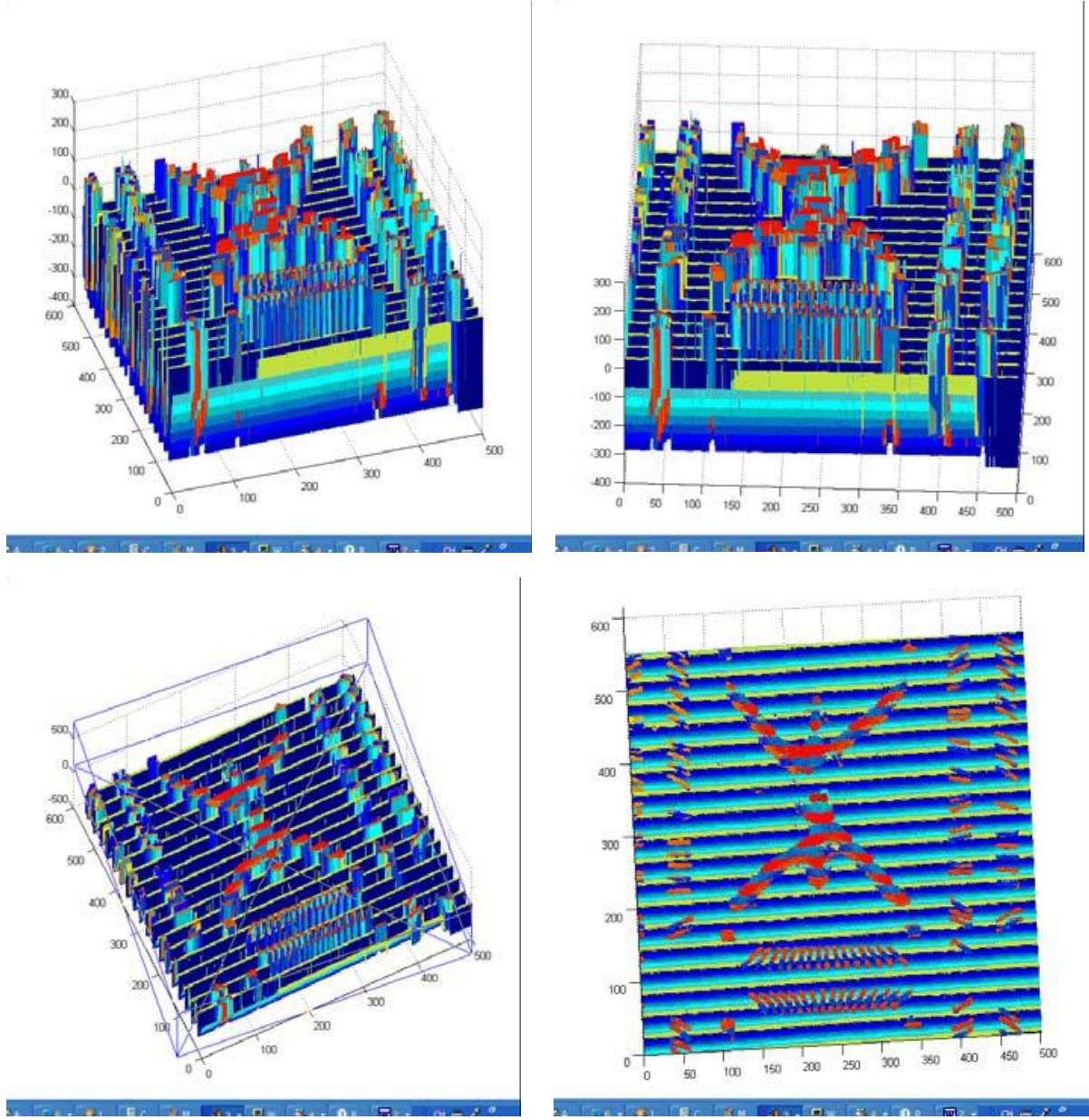

Fig. 7. 3D reconstruction of the inspected surface by the proposed method, as observed from different angles.

\section{REFERENCES}

[1] D. Bergmann, "New approach for automatic surface reconstruction with coded light", SPIE 2572, pp 2-9, 1995

[2] M. Halioua and H.C. Liu, "Optical Sensing Techniques for 3-D Machine Vision”, SPIE 665, pp 150-161, 1986

[3] O. Hall-Holt, S. Rusinkiewicz, "stripe boundary Codes for real-time structured-light range scanning of moving objects", ICCV, pp 359-366, 2001

[4] M. Ishihara, "High-speed 3D shape measurement using a nonscanning multiple-beam confocal imaging system", SPIE 3478, pp 68-75, 1998

[5] B. Liu, L. Yang, and J. Zhang, "Laser scanning technique for 3D measurement", SPIE 3415, pp 95-98, 1998

[6] Y. Matsuyama, T. Honda, H. Yamamura, H. Sasazawa, M. Nomoto, T. Ninomiya, A. Schick, L. Listl, P. Kollensperger, D. Spriegel, P. Mengel, and R. Schneider, "Automated solder joint inspection system using optical 3-D image detection", Applications of Computer Vision, 1996, Proceedings 3rd IEEE Workshop on, pp116-122, 1996

[7] T. Ode, "A new technique for optical 3D measurements with a confocal scanning laser microscope", Instrumentation and Measurement Technology Conference, 1994, pp 672-676, 1994

[8] J. L. Posdamer and M.D. Altschuler, "Surface measurement by space-encoded projected beam systems", Computer Graphics and Image Processing, 18 (1), pp 1-17, 1982 
[9] C. Quan, C.J. Tay, X.Y. Kang, and H.M. Shang, "Microscopic surface contouring by fringe projection method", Optics Laser Technology, 34, pp 547-552, 2002

[10] A.R. Rao, N. Ramesh, F.Y. Wu, J.R. Mandeville, and P.J.M. Kerstens, "Algorithms for a fast confocal optical inspection system", Applications of Computer Vision, Proceedings, 1992., IEEE Workshop on, pp 298-305, 1992

[11] J. Salvi, J. Pags, and J. Batlle, "Pattern codification strategies in structured light systems", Pattern Recognition, 37(4), pp 827-849, 2004

[12] A. Schick and M. Kedziora, "Inspection and process evaluation for flip chip bumping and CSP by scanning 3D confocal microscopy", Advanced Packaging Materials, 2002. Proceedings, pp 116-119, 2002

[13] H. Stern, "Laser based 3-D surface mapping for manufacturing diagnostics and reserve engineering", Aerospace and Electronics Conference, Proceedings of the IEEE, pp 1205-1212, 1992

[14] S.Q. Wang, B.H. Zhuang, and W.W. Zhang, "New principle of optical displacement measurement based on light scattering from rough surface", SPIE 2909, pp 37-42, 1997 